\title{
Cryptosystems using an improving hiding technique based on latin square and magic square
}

\author{
Sahab Dheyaa Mohammed ${ }^{1}$, Taha Mohammed Hasan ${ }^{2}$ \\ ${ }^{1}$ University of Information Technology and Communications, Iraq \\ ${ }^{2}$ University of Diyala, College of Science, Iraq
}

\begin{tabular}{l} 
Article Info \\
\hline Article history: \\
Received Feb 5, 2020 \\
Revised Apr 7, 2020 \\
Accepted Apr 19, 2020 \\
\hline
\end{tabular}

Keywords:

Affine cipher function Encryption process

Finite fields

Irreducible polynomial

Latin square

\begin{abstract}
Hackers should be prevented from disclosing sensitive data when sent from one device to another over the network. Therefore, the proposed method was established to prevent the attackers from exploiting the vulnerabilities of the redundancy in the ciphertext and enhances the substitution and permutation operations of the encryption process the solution was performed by eliminates these duplicates by hiding the ciphertext into a submatrix $4 \times 4$ that chooses randomly from magic square $16 \times 16$ in each ciphering process. Two techniques of encrypted and hiding were executed in the encryption stage by using a magic square size $3 \times 3$ and Latin square size $3 \times 3$ to providing more permutation and also to ensure an inverse matrix of decryption operation be available. In the hiding stage, the ciphertext was hidden into a $16 \times 16$ matrix that includes 16 sub-magic squares to eliminate the duplicates in the ciphertext. Where, all elements that uses were polynomial numbers of a finite field of degree Galois Fields GF $\left(2^{8}\right)$. The proposed technique is robust against disclosing the repetition encrypted data based on the result of Avalanche Effect in an accepted ratio (62\%) and the results of the output of the proposed encryption method have acceptable randomness based on the results of the p-values (0.629515) of the National Institute of Standards and Technology (NIST) randomness tests. The work can be considered significant in the field of encrypting databases because the repetition of encrypted data inside databases is considered an important vulnerability that helps to guess the plaintext from the encrypted text.
\end{abstract}

Copyright $\Subset 2020$ Institute of Advanced Engineering and Science. All rights reserved.

\section{Corresponding Author:}

Sahab Dheyaa Mohammed,

University of Information Technology and Communications,

Baghdad, Iraq.

Email:sahab7dia@gmail.com

\section{INTRODUCTION}

Encryption that uses a peculiar number system is a good method to encode and decode data and provides additional security against attacks during transmission and providing opportunities for full encryption and decryption whilst hiding all technical details [1]. Numerous techniques are used to secure file transfer, including the types of encryption techniques designed to keep files secure. Substitution and transposition are two mechanisms used in symmetric encryption. Substitution involves changing plain text values to cipher text values. By contrast, the transposition moves the locations of plain text values [2].

Nowadays, attackers are trying to break the encryption algorithm by retrieving the key, or by analyzing a collision or the existence of repeated bits / characters (bytes) in the encrypted message to gain the algorithm of encryption or the key utilized for it. Therefore, the encryption method must be efficient and exclude repeated terms and the attacker cannot track the repetition [3]. The traditional cryptographic algorithm suffers from the problem of data redundancy in the ciphertext. This proposed method aims to develop an algorithm to exclude redundancy in the ciphertext. Where, new encryption and hiding algorithms 
are implemented, using magic square and Latin square to increase the permutation and substitution to make encryption more secure and complex. In multiple encryption methods, many mathematical models and operations are used to improving encryption methods such as matrix multiplication, magic square, and Latin square. In this paper, we used the magic square and Latin square to improve the proposed encryption method and hide encrypted data.

\subsection{Magic square and latin square}

A magic square is a square matrix of integers with the same sum of the values in the rows, columns and main diagonals. As shown in Figure 1, a magic square of the fourth-order (i.e., $4 \times 4$ ) has a magic sum of 24 , which is the total sum of the values in the rows, columns and main diagonals. A total of 880 different fourth-order magic squares are provided [4]. A Latin square is an $n \times n$ array of order $n$, in which all rows and columns contain $\{0,1,2 \ldots n-1\}$ precisely once and the also the symbols occur precisely once in each row and column. A Latin square is called diagonalise.

Latin square when the square has a main diagonal in a transversal form. Latin squares can be constructed using number theory using only one step from the magic square, particularly by using the modulo $\mathrm{n}$ of the magic square. In this manner, the Latin square obtained are of two types, namely, diagonalised and doubly diagonalised Latin squares of any odd order $n$ [5]. That is, a magic square is an $n \times n$ array with integers $\left\{0 ; 1 \ldots n^{2}-1\right\}$, such that, each number is filled once in each row and column and the sum of each row, column and main diagonal or main antidiagonal is the same constant value. An example for order 3 as in Figure $2[6]$.

\begin{tabular}{|c|c|c|c|}
\hline 1 & 2 & 16 & 15 \\
\hline 13 & 14 & 4 & 3 \\
\hline 12 & 7 & 9 & 6 \\
\hline 8 & 11 & 5 & 10 \\
\hline
\end{tabular}

Figure 1. The magic square $(4 \times 4)[4]$

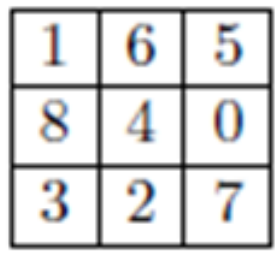

Figure 2. Order 3 of magic square [6]

A Latin square design is an approach of mapping elements to appear in an equal form into a square matrix. Elements appear one time in each row and column. The processes are assigned at random into the square, with each process appearing one time per row and column [7]. No algorithm is used to build all kinds of magic square. Thus, the algorithm that executes for even squares is different from the algorithm that works on odd order.

There are three methods for constructing magic squares according to the matrix dimensions are those of the odd order, singly even order and doubly even order [8]. Magic Squares of the Odd Order a simple type of magic square is of the form $2 \mathrm{~m}+1$, where $\mathrm{m}$ is a positive integer. The De la Loubère's method is an example of an odd order, in which the matrix size may be $3 \times 3,5 \times 5$, and $7 \times 7$, amongst others.

Odd order magic squares were builded by using different methods, such as the pyramid, de la Loubere's, or staircase method [9]. Magic Squares of a Doubly Even Order the order of the doubly even ordered squares is of the form $4 \mathrm{n}$ (e.g., $4,8, \ldots$ ) or may be divided by 2 and 4 . An example is the method developed by Albrecht Dürer. The size of the square matrix is $4 \times 4,8 \times 8$ and $12 \times 12$, amongst others [10]. Magic Squares of a Singly Even Order. Singly even square in the order $n$ is of the form $2(2 \mathrm{~m}+1)=4 \mathrm{n}+2(\mathrm{e} . \mathrm{g}$., $2,6,10,14,18,22$, amongst others). The order can be divided by 2 but not 4 . An example of this order is Philippe de la Hire's method. The size of the matrix is $6 \times 6,10 \times 10$ and $14 \times 14$, amongst others [11].

Different approaches used to construct magic squares have been developed during the past years. An example is the dotting method, which depends on cells marked by dots for the magic square. To construct a $4 \times 4$ magic square, dots are first placed on the main diagonals. Thereafter, the cells are computed from a corner and numbers are written in every marked cell. When the last cell is reached, the cells are reviewed in reverse and the numbers are placed sequentially in each cell without dots. Figure 3 illustrates the arranged dots in the square. The magic square $(12 \times 12)$ is obtaiend via computing the cells starting from a corner and ending in the opposite corner see Figure 4 [12]. All mathematical operations used in the proposed method were performed on a polynomial numbers of degrees GF $\left(2^{8}\right)$. 


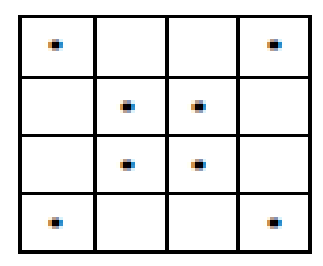

Figure 3. Arranged dots in the square

\begin{tabular}{|c|c|c|c|c|c|c|c|c|c|c|c|}
\hline 1 & 143 & 142 & 4 & 5 & 139 & 138 & 8 & 9 & 135 & 134 & 12 \\
\hline 132 & 14 & 15 & 129 & 128 & 18 & 19 & 125 & 124 & 22 & 23 & 121 \\
\hline 120 & 26 & 27 & 117 & 116 & 30 & 31 & 113 & 112 & 34 & 35 & 109 \\
\hline 37 & 107 & 106 & 40 & 41 & 103 & 102 & 44 & 45 & 99 & 98 & 48 \\
\hline 49 & 95 & 94 & 52 & 53 & 91 & 90 & 56 & 57 & 87 & 86 & 60 \\
\hline 84 & 62 & 63 & 81 & 80 & 66 & 67 & 77 & 76 & 70 & 71 & 73 \\
\hline 72 & 74 & 75 & 69 & 68 & 78 & 79 & 65 & 64 & 82 & 83 & 61 \\
\hline 85 & 59 & 58 & 88 & 89 & 55 & 54 & 92 & 93 & 51 & 50 & 96 \\
\hline 97 & 47 & 46 & 100 & 101 & 43 & 42 & 104 & 105 & 39 & 35 & 108 \\
\hline 36 & 110 & 111 & 33 & 32 & 114 & 115 & 29 & 28 & 118 & 119 & 25 \\
\hline 24 & 122 & 123 & 21 & 20 & 126 & 127 & 17 & 16 & 130 & 131 & 13 \\
\hline 133 & 11 & 10 & 136 & 137 & 7 & 6 & 140 & 141 & 3 & 2 & 144 \\
\hline
\end{tabular}

Figure 4. Constructed magic square $(12 \times 12)$ [12]

\subsection{Finite fields}

Finite fields are a collection of finite elements also called Galois fields (GF), which was named after Evariste Galois (1811-1832). Galois studied the scope of polynomials and discovered many of their principles. Numerous applications have used finite fields, such as cryptographic algorithms (Diffie and Hellman, 1976; ElGamal, 1985; Miller, 1986; Kravitz, 1993) and advanced encryption standard (AES) [13].

The finite fields executed for the order $\mathrm{F}_{2}^{\mathrm{n}}$ is defined as $\mathrm{GF}\left(2^{\mathrm{n}}\right)$ as a set of $2^{\mathrm{n}}$ elements. The two binary operations + and $\times$ are defined in this set. Each nonzero element of the field has a multiplicative inverse [14]. All operations of finite fields produce an element in the field arrangement based on the $p^{n}, \mathrm{p}$ indicates a prime number and $\mathrm{n}$ indicates a positive integer [15]. One of the cases in finite fields is when the prime $(p)=2$, in which the elements of GF $\left(2^{n}\right)$ are expressed as binary numbers. One of the uses of GF $\left(2^{n}\right)$ is a polynomial. $f(x)$ of polynomial number in GF $\left(2^{n}\right)$ is showed in (1), which can be represented uniquely as $n$ binary coefficients $\left(a_{n-1} a_{n-2} \ldots a_{0}\right)[14]$.

$$
f(x)=a_{n-1} x^{n-1}+a_{n-2} x^{n-2}+\cdots+a_{1} x+a_{0}=\sum_{i=0}^{n-1} a_{i} x^{i} \cdots
$$

Finite field multiplication represents multiplying two polynomials elements and sums them like powers of $\mathrm{x}$ as a result. When multiplication result greater than $\mathrm{n}-1$, so, the result is minimized via the module of irreducible polynomial $\mathrm{m}(\mathrm{x})$ of grade $\mathrm{n}$ then the result divided by $m(x)$ and kept the remainder [14]. "An irreducible polynomial is a polynomial $f(x)$ over a field GF $\left(2^{n}\right)$, if and only if $f(x)$ can not be expressed as a two polynomials product, both over GF $\left(2^{n}\right)$ and both of degree lower than of $f(x)$ ". Moreover, a polynomial is irreducible when it is divisible by itself and 1 (without remainder) $[14,16]$.

\subsection{Related works}

a) Dharini (2014) proposed the encryption methods RSA for secure data transmission, in which SSL over RSA and combined magic square provide additional security to the system; moreover, the confidentiality and integrity of data sent to and from the cloud are ensured [17].

b) Chenglian Liu (2011) proposed a novel approach of streams cipher application for random access file that can be easily implemented according to the magic square method, also improve the model of the cipher stream to strengthen the protection efficiently and has a high speed of key stream generator [18]. 
c) Shahla (2017) proposed an approach to creating a magic square of order 32, which represents the difficulty in tracing this square in the cryptography and improving efficiency by providing robust security to the encryption. The magic squares have numerous random numbers rather than the ASCII values and are used to generate the keys of the public-key encryption algorithms [19].

d) Authors (2013) this paper aim to enhance an algorithm to eliminate the repetitive characters or symbols in the ciphertext by using extra algorithms such as Function Encryption, NJJSAA, Bit Rotation, and Reverse method, the encryption is very difficult to break and more secure [3].

e) Ako (2016) the author has proposed a new method that combines encryption and information hiding to increase security, privacy, accuracy, and confidentiality. A hash least significant bit method has been suggested for the hiding encryption data process with the use of an affine cipher to provide more encryption and increase data security in the network environment [20].

f) Authors (2016). proposed approach is used that combines the encryption method with a method of hiding encrypted data to increase the security and maintain confidentiality, integrity, and availability of data against external attacks and unauthorized access. The plain text was encrypted with the RSA algorithm and the ciphertext was hidden into the image using the advanced LSB method. Where the plain text was encoded and divided into parts $\mathrm{P} 1$ and $\mathrm{P} 2$, the XOR operation was performed on the part (P1) of the odd locations and (p2) using even location for LSB+1 [21].

g) Jeena Pappachan, Jinu Baby (2015), suggested one of the kinds of chaotic maps (Tinkerbell Maps) with the magic square encrypt the images. The proposed method provides efficiency and security for encryption images.the proposed method consists of a 128-key secret key or a 16-character hexadecimal key that divides into 16 8-bit subkeys. The magic squares and two-dimensional maps are created, row shifting, pixel adjustment [22].

h) The authors (2012) proposed encryption grayscale and color images method using a symmetric-key Latin square image cipher (LSIC). this method improved novel Latin square image encryption and a novel method of merging probabilistic encryption in image encryption by including random noise by LSB technique. The proposed LSIC has a secure cipher due to large keyspace, excellent confusion, and diffusion approach and powerful against channel noise and brute-force attacks [23].

i) Al-Hasan (2018), proposed a new steganography approach is proposed by converting the cover image from RGB color space to $\mathrm{YCbCr}$ color space. Then, hide the encrypted data using the Affine Cipher and Magic Square Matrix are applied to embed the encrypted data onto the cover image using the ISB approach. Then the salt-and-pepper noise is added to the cover image. The results show that the proposed method withstands against attacks [24].

j) Tomba I (2017), an improved cryptosystem by uses 5 pseudo letters $\{\mathrm{Au}, \mathrm{Ea}, \mathrm{Ee}, \mathrm{Oo}, \mathrm{Ou}\}$ in the sequence of 26 English letters. The proposed pseudo letters are using magic squares or any type of matrices in encryption and decryption operation. Using pseudo letters will affect the ASCII characteristics thereby will provide an additional layer of security of the improved cryptosystem [25].

\section{PROPOSED METHODOLOGY}

The proposed encryption method contributes to security enhancement and eliminates the symbols repetition of ciphertext by using many new methods such as magic square, Latin square to encryption data and constructing random magic square to hiding ciphertext inside it, which provides additional safety features. The majority of the encryption methods suffer from the repetition of the elements into the ciphertext. So, random magic squares were used to hiding the repetition in the ciphertext. In the proposed method, the encryption and decryption processes were executed in two phases. The first phase is done by using a $3 \times 3$ Latin square derived from the odd ordered $3 \times 3$ magic square to arrange the 9 key elements of the polynomial numbers of degree GF $\left(2^{8}\right)$. the plaintext of the 9-byte polynomial numbers is arranged in odd ordered $3 \times 3$ magic square and multiplying with Latin square $3 \times 3$ using the operations of finite fields with an irreducible polynomial of GF $\left(2^{8}\right)$. The second phase involves constructs magic square of a doubly even order $12 \times 12$ includes 16 sub-squares of even magic square to hide the encrypted text of the first phase randomly. Figure 5 presents the block diagram of the encryption operation. The coding scheme alone does not provide sufficient security. Thus, magic square provides the permutation of the character encoding based on the magic square schemes.

\subsection{Encoding}

All plain text were labeled using the ASCII code for coding the letters to polynomial numbers of GF $\left(2^{8}\right)$. Thereafter, the numbers were arranged in $3 \times 3$ matrix [PO] based on podision. Then the encoded numbers of plaintext are arrang in a 33 matrix [p] based on the construction of the odd order $3 \times 3$ magic square (M).

$$
[\mathrm{PO}] \rightarrow[\mathrm{P}]
$$




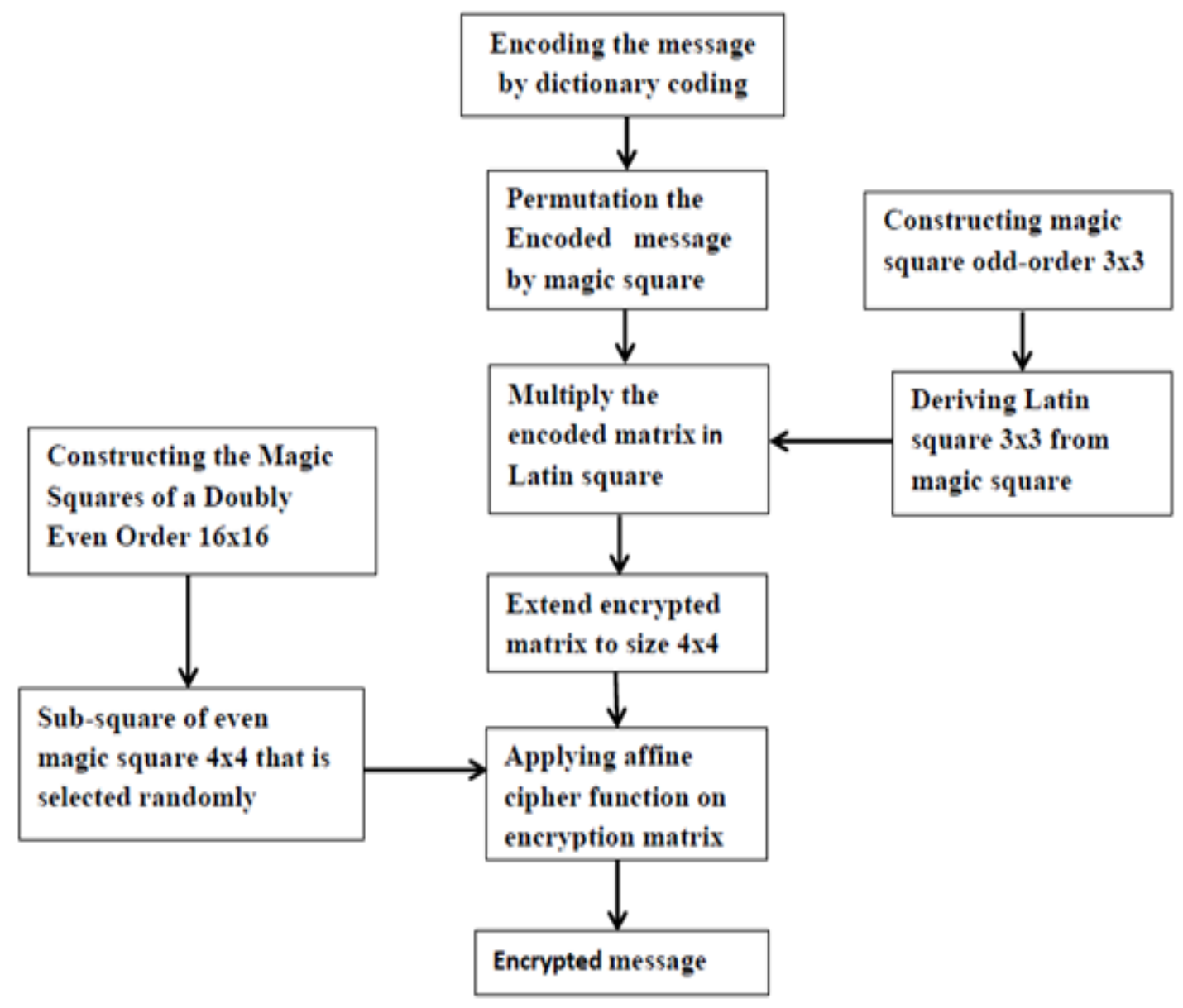

Figure 5. The block diagram of encryption operation

\subsection{Encryption}

The encryption process includes two phases of encryption and hiding the encrypted text.

Phase 1: The encoded elements of the plain text were arranged in a $3 \times 3$ matrix $[p]$, and the key elements are arranged in an odd order $3 \times 3$ magic square $[\mathrm{M}]$. Thereafter, the $3 \times 3$ Latin square $[L]$ was derived from the magic square by taking modulo 3 as (2). The encrypted text was executed by multiplying the $3 \times 3$ Latin square $[L]$ and encoded matrix $[P]$ with an irreducible polynomial $(m)\left(x^{8}+x^{4}+x^{3}+x+1\right)$ as (3).

$$
\begin{aligned}
& L_{i}=M_{i} \bmod 3 \\
& {[C]=([L] .[P]) \bmod m}
\end{aligned}
$$

where

[C]: The encrypted matrix $3 \times 3$

Phase 2: The encrypted matrix [C] of the previous stage was randomly hidden inside the magic square [MS] of the $12 \times 12$ doubly even order. [MS] was divided into 16 by sub-magic square $4 \times 4$. The encrypted matrix [C] resulting from the previous stage is expanded to a $4 \times 4$ by randomly adding 8 numbers (salt) from 1 to 255 (polynomial numbers of GF $\left(2^{8}\right)$ ). Thereafter, the matrix [C] was imultiplied with one of the sub-magic squares [A] (selected randomly) by the affine cipher function. Each element of the encryption matrix $C_{i}$ corresponds to one element in the sub-magic square $A_{i}$. The affine cipher as in (4) was applied, except one element that is left as a pointer. The result is a matrix [E] $4 \times 4$ represents the final of cipher text.

$$
E_{i}=\left(A_{i} \cdot C_{i}+b\right) \operatorname{Mod} m
$$


The Encryption algorithm of proposed method as the following:

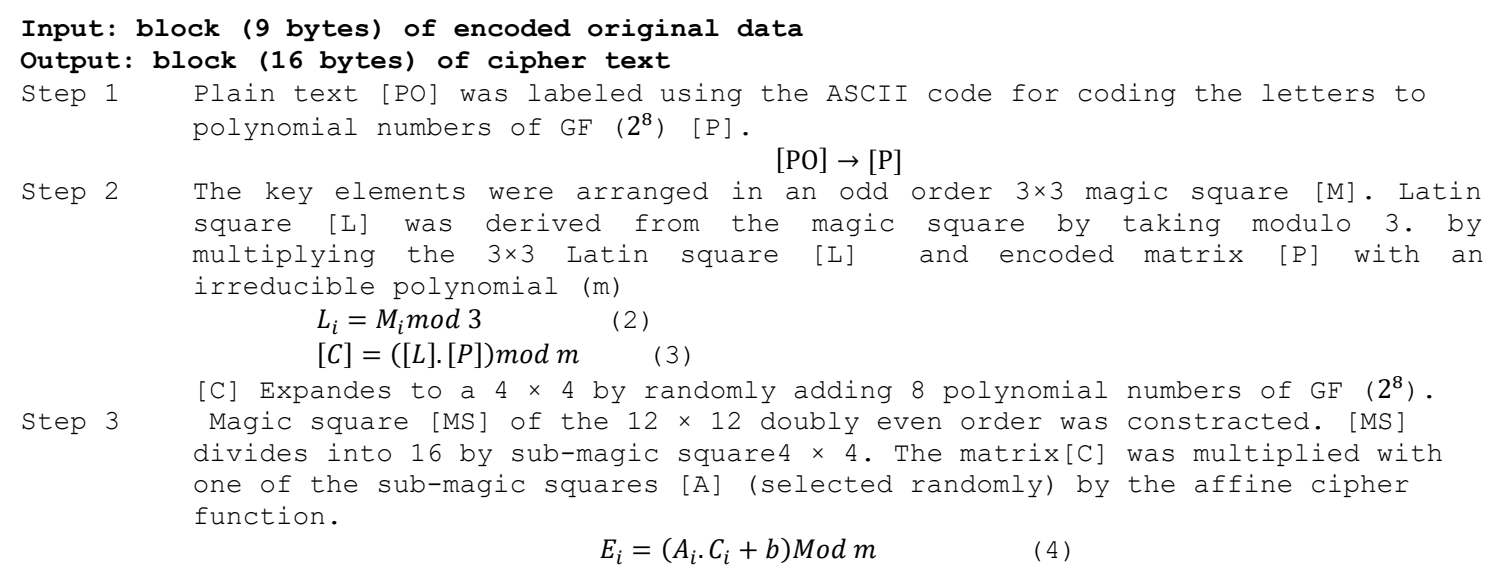

\subsection{Decryption}

The decryption process was executed by reversing the previous stages of the encryption process.

Phase 1: In this phase, the encryption matrix [c] was returned by (5), where uses the inverse of each element of the specific sub-matrix [A] multiply with final encryption matrix elements [E]. The sub-magic square [A] was selected using the element (pointer) into the matrix. Then, the encryption matrix [C] was reduced from $4 \times 4$ to $3 \times 3$ by eliminating the random numbers (salt).

$$
[C]=A_{i}^{-1}\left(E_{i}-b\right) \bmod m
$$

Phase 2: This stage uses the inverse of $3 \times 3$ Latin squares $\left[L^{-1}\right]$ and multiplied by the cipher matrix [C] to obtain the encoded plain text matrix [P]as in (6).

$$
[P]=\left(\left[L^{-1}\right] \cdot[C]\right) \bmod m
$$

Phase 3: Decoding the encoded plaintext matrix [P] to the plain text matrix $3 \times 3$ [PO] based on the positions of magic square elements $[\mathrm{M}]$.

$$
[P] \rightarrow[P O]
$$

The Decryption algorithm of proposed method as the following:

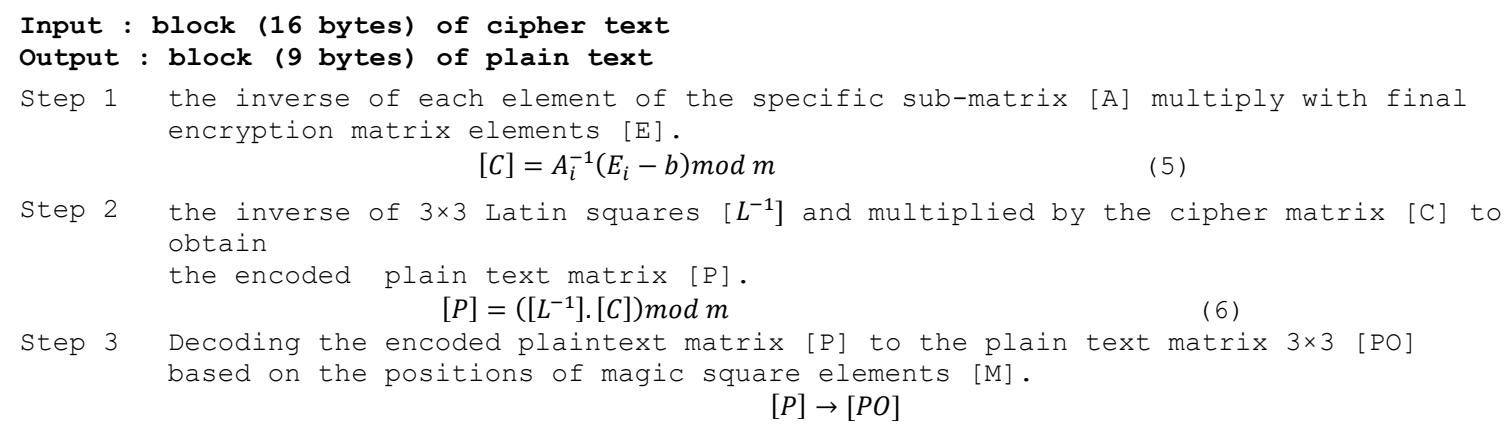

\section{Example}

The message to be sent is assumed to be "Ciphering" and the key is a magic square " $35,37,39,41$, 43, 45, 47, 49 and 51', the steps of the encryption process as the follows:

a) The message of the plain text is encoded using the ASCII code as a Figure 6. 


\begin{tabular}{|c|c|c|c|c|c|c|c|c|c|}
\hline plaintext & c & i & p & h & e & r & i & n & g \\
\hline cod & 99 & 105 & 112 & 104 & 101 & 114 & 105 & 110 & 103 \\
\hline position & 1 & 2 & 3 & 4 & 5 & 6 & 7 & 8 & 9 \\
\hline
\end{tabular}

Figure 6. Encoding the message

b) One odd-order $3 \times 3$ magic square $(\mathrm{M})$ is constructed for the permutation of the elements of the plain text based on the position of elements in $(\mathrm{M})$, thereby obtaining the encoded matrix $(\mathrm{P})$.

Plaintext position

\begin{tabular}{|l|l|l|}
\hline $\mathrm{c}$ & $\mathrm{i}$ & $\mathrm{p}$ \\
\hline $\mathrm{h}$ & $\mathrm{e}$ & $\mathrm{r}$ \\
\hline $\mathrm{i}$ & $\mathrm{n}$ & $\mathrm{g}$ \\
\hline
\end{tabular}$\rightarrow$\begin{tabular}{|l|l|l|}
\hline 1 & 2 & 3 \\
\hline 4 & 5 & 6 \\
\hline 7 & 8 & 9 \\
\hline
\end{tabular}

[M]

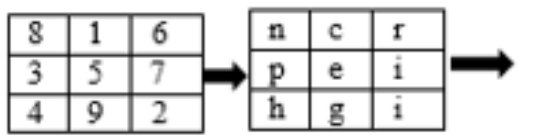

Encoded matrix [P]

\begin{tabular}{|l|l|l|}
\hline 110 & 99 & 114 \\
\hline 112 & 101 & 105 \\
\hline 104 & 109 & 105 \\
\hline
\end{tabular}

c) An odd order $3 \times 3$ magic square is constructed and a $3 \times 3$ Latin square (L) is derived from the MS base on mod 3 , and multiplied in the encoded matrix with irreducible polynomial $\left(x^{8}+x^{4}+x^{3}+x+1\right)$ as (7) and (8).

$$
L_{i}=M_{i} \bmod 3
$$

[M]

\begin{tabular}{|l|l|l|}
\hline 49 & 35 & 45 \\
\hline 39 & 43 & 47 \\
\hline 41 & 51 & 37 \\
\hline
\end{tabular}

$\operatorname{Mod} 3=$

$$
[C]=([L] \cdot[P]) \bmod m
$$

\section{[L]}

\begin{tabular}{|l|l|l|}
\hline 1 & 2 & 0 \\
\hline 0 & 1 & 2 \\
\hline 2 & 0 & 1 \\
\hline
\end{tabular}

[ P]

\begin{tabular}{|l|l|l|}
\hline 110 & 99 & 114 \\
\hline 112 & 101 & 107 \\
\hline 104 & 109 & 105 \\
\hline
\end{tabular}

[L]

\begin{tabular}{|l|l|l|}
\hline 1 & 2 & 0 \\
\hline 0 & 1 & 2 \\
\hline 2 & 0 & 1 \\
\hline
\end{tabular}

d) The encrypted matrix is extended to $4 \mathrm{x} 4(\mathrm{Cx})$ by randomly adding 8 polynomial numbers of $\mathrm{GF}\left(2^{8}\right)$.

$\mathbf{C X}=\quad$\begin{tabular}{|l|l|l|l|}
\hline $\mathbf{2 4 3}$ & 142 & 169 & 160 \\
\hline $\mathbf{2 3 1}$ & 160 & 191 & 187 \\
\hline $\mathbf{1 5 0}$ & 180 & 171 & 141 \\
\hline 12 & 7 & 122 & 199 \\
\hline
\end{tabular}

e) Building The Magic Square doubly even order 16×16 including 16 sub-square of even magic square is as in Figure 7. 


\begin{tabular}{|c|c|c|c|c|c|c|c|c|c|c|c|c|c|c|c|}
\hline 1 & 255 & 254 & 4 & 5 & 251 & 250 & 8 & 9 & 247 & 246 & 12 & 13 & 243 & 242 & 16 \\
\hline 240 & 18 & 19 & 237 & 236 & 22 & 23 & 233 & 232 & 26 & 27 & 229 & 228 & 30 & 31 & 225 \\
\hline 224 & 34 & 35 & 221 & 220 & 38 & 39 & 217 & 216 & 42 & 43 & 213 & 212 & 46 & 47 & 209 \\
\hline 49 & 207 & 206 & 52 & 53 & 203 & 202 & 56 & 57 & 199 & 198 & 60 & 61 & 195 & 194 & 64 \\
\hline 65 & 191 & 190 & 68 & 69 & 187 & 186 & 72 & 73 & 183 & 182 & 76 & 77 & 179 & 178 & 80 \\
\hline 176 & 82 & 83 & 173 & 172 & 86 & 87 & 169 & 168 & 90 & 91 & 165 & 164 & 94 & 95 & 161 \\
\hline 160 & 98 & 99 & 157 & 156 & 102 & 103 & 153 & 152 & 106 & 107 & 149 & 148 & 110 & 111 & 145 \\
\hline 113 & 143 & 142 & 116 & 117 & 139 & 138 & 120 & 121 & 135 & 134 & 124 & 125 & 131 & 130 & 128 \\
\hline 129 & 127 & 126 & 132 & 133 & 123 & 122 & 136 & 137 & 119 & 118 & 140 & 141 & 115 & 114 & 144 \\
\hline 112 & 146 & 147 & 109 & 108 & 150 & 151 & 105 & 104 & 154 & 155 & 101 & 100 & 158 & 159 & 97 \\
\hline 96 & 162 & 163 & 93 & 92 & 166 & 167 & 89 & 88 & 170 & 171 & 85 & 84 & 174 & 175 & 81 \\
\hline 177 & 79 & 78 & 180 & 181 & 75 & 74 & 184 & 185 & 71 & 70 & 188 & 189 & 67 & 66 & 192 \\
\hline 193 & 63 & 62 & 196 & 197 & 59 & 58 & 200 & 201 & 55 & 54 & 204 & 205 & 51 & 50 & 208 \\
\hline 48 & 210 & 211 & 45 & 44 & 214 & 215 & 41 & 40 & 218 & 219 & 37 & 36 & 222 & 223 & 33 \\
\hline 32 & 226 & 227 & 29 & 28 & 230 & 231 & 25 & 24 & 234 & 235 & 21 & 20 & 238 & 239 & 17 \\
\hline 241 & 15 & 14 & 244 & 245 & 11 & 10 & 248 & 249 & 7 & 6 & 252 & 253 & 3 & 2 & 256 \\
\hline
\end{tabular}

Figure 7. Magic square doubly even order $16 \times 16$

f) By applying the affine cipher function on encryption matrix (C) and sub-square of the even $4 \times 4$ magic square [A] selected randomly, the result represents the final encrypted matrix. The affine cipher function on each element of the encryption matrix is applied, except the element at the bottom of the left corner is substituted with an element at the bottom left corner in the sub-magic square to be a pointer, where (b) value is equal 180 as a constant in (9).

$$
E_{i}=\left(A_{i} \cdot C x_{i}+b\right) M o d m
$$

\begin{tabular}{|l|l|l|l|}
\hline 1 & 255 & 254 & 4 \\
\hline 240 & 18 & 19 & 237 \\
\hline 224 & 34 & 35 & 221 \\
\hline 49 & 207 & 206 & 52 \\
\hline
\end{tabular}

$\mathbf{C x}=$\begin{tabular}{|l|l|l|l|}
\hline $\mathbf{2 4 3}$ & 142 & 169 & 160 \\
\hline $\mathbf{2 3 1}$ & 160 & 191 & 187 \\
\hline $\mathbf{1 5 0}$ & 180 & 171 & 141 \\
\hline 12 & 7 & $\mathbf{1 2 2}$ & 199 \\
\hline
\end{tabular}

$\mathrm{E}=$\begin{tabular}{|l|l|l|l|}
\hline 71 & 92 & 236 & 2 \\
\hline 141 & 1 & 107 & 225 \\
\hline 60 & 182 & 238 & 87 \\
\hline 227 & 239 & 174 & 52 \\
\hline
\end{tabular}

\subsection{The decryption process uses two stages as follows:}

g) The inverse of the affine cipher function on the encryption matrix (E) and the specific sub-inverse magic square affine cipher is applied as following in inverse (10):

$$
C x=A^{-1}(E-b) \bmod m
$$

$A^{-1}=$\begin{tabular}{|l|l|l|l|}
\hline 1 & 28 & 65 & 203 \\
\hline 91 & 170 & 75 & 80 \\
\hline 177 & 90 & 241 & 248 \\
\hline 69 & 230 & 172 & 52 \\
\hline
\end{tabular}$\quad \mathbf{E}=$\begin{tabular}{|l|l|l|l|l|}
\hline $\mathbf{7 1}$ & 92 & $\mathbf{2 3 6}$ & $\mathbf{2}$ \\
\hline $\mathbf{1 4 1}$ & $\mathbf{1}$ & $\mathbf{1 0 7}$ & $\mathbf{2 2 5}$ \\
\hline $\mathbf{6 0}$ & $\mathbf{1 8 2}$ & $\mathbf{2 3 8}$ & $\mathbf{8 7}$ \\
\hline $\mathbf{2 2 7}$ & $\mathbf{2 3 9}$ & $\mathbf{1 7 4}$ & $\mathbf{5 2}$ \\
\hline
\end{tabular}$\quad \mathbf{C x}=$\begin{tabular}{|l|l|l|l|l|}
$\mathbf{2 4 3}$ & 142 & $\mathbf{1 6 9}$ & $\mathbf{1 6 0}$ \\
\hline $\mathbf{2 3 1}$ & 160 & 191 & $\mathbf{1 8 7}$ \\
\hline $\mathbf{1 5 0}$ & 180 & $\mathbf{1 7 1}$ & $\mathbf{1 4 1}$ \\
\hline $\mathbf{1 2}$ & $\mathbf{7}$ & $\mathbf{1 2 2}$ & $\mathbf{1 9 9}$ \\
\hline
\end{tabular}

h) The matrix is returned to $3 \times 3$ [c] and the random numbers (salt) that added at the encryption stage were deleted. 


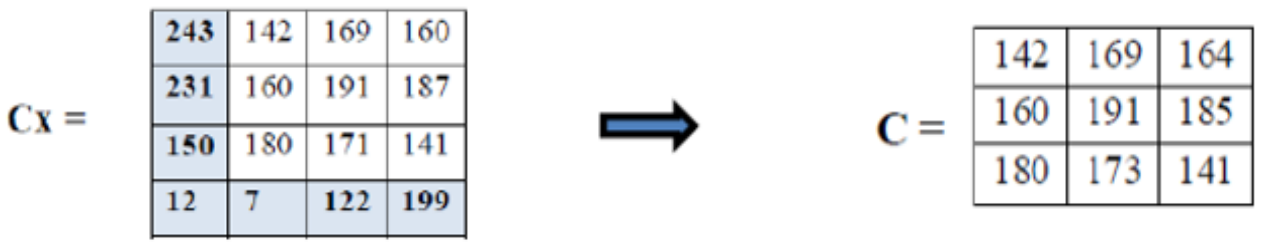

i) The inverse of the Latin square is used and multiplied with the $3 \times 3$ encryption matrix as following in (11).

$$
P=\left(\left[L^{-1}\right] .[C]\right) \operatorname{Mod} m
$$

$L^{-1}=$\begin{tabular}{|l|l|l|}
\hline 79 & 158 & 39 \\
\hline 39 & 79 & 158 \\
\hline 158 & 39 & 79 \\
\hline
\end{tabular}

$$
\mathbf{C}=\begin{array}{|l|l|l|}
\hline 142 & 169 & 164 \\
\hline 160 & 191 & 185 \\
\hline 180 & 173 & 141 \\
\hline
\end{array}
$$

$P=$\begin{tabular}{|l|l|l|}
\hline 110 & 99 & 114 \\
\hline 112 & 101 & 107 \\
\hline 104 & 109 & 105 \\
\hline
\end{tabular}

\section{RESULTS AND ANALYSIS}

The proposed method provided the processing of a repetition problem in the ciphertext. The important characteristic of the encryption algorithm is the Avalanche Effect, where any single bit change in the plain text or key must be a more change in the bits of encrypted text. Avalanche effect test of the proposed method can be calculated by using (12). The test calculates the avalanche effect of the proposed method when changing 1-bit in the key and keeping plaintext constant and changing in plaintext by 1-bit and keeping the Key bits constant. In two cases, the number of bits that differ between the two cipher-texts is calculated by XOR operation. Then, Table 1 shows the avalanche effect of proposed method:

$$
\text { Avalanche Effect }=\frac{\text { Number of chang bits in the ciphered text }}{\text { Number of bits in the ciphered text }} \times 100 \%
$$

\begin{tabular}{|c|c|c|c|c|c|}
\hline $\begin{array}{c}\text { Sample } \\
\text { No. }\end{array}$ & Keys & input & Output MDES & $\begin{array}{l}\text { Avalan } \\
\text { che test }\end{array}$ & $\begin{array}{c}\text { Avalanche } \\
\text { Test } \% \\
\end{array}$ \\
\hline 1 & $\begin{array}{l}49,35,45,39,43,47, \\
41,51,37 \\
49,35,45,39, \mathbf{1 7 1}, 47, \\
41,51,37\end{array}$ & $\begin{array}{l}99,105,112,104,101 \\
114,105,110,103 \\
99,105,112,104,101 \\
114,105,110,103\end{array}$ & $\begin{array}{l}71,92,236,2,141,1,107,225,60, \\
182,238,87,227,239,174,52 \\
255,242,24,111,282,255,158,62, \\
210,32,161,180,250,49,251,171\end{array}$ & 0.648 & $64.8 \%$ \\
\hline 2 & $\begin{array}{l}18,155,80,228,110 \\
160,14,51,169 \\
18,155,80,228,110 \\
160,14,51,169\end{array}$ & $\begin{array}{l}67,111,109,112,117 \\
116,101,114,115 \\
67,111,109, \mathbf{2 4 0}, 117 \\
116,101,114,115\end{array}$ & $\begin{array}{l}108,157,124,219,140,170,151,191, \\
20,113,224,181,184,253,200,70 \\
156,105,243,139,233,217,254,67 \\
250,191,26,90,150,1,50,212\end{array}$ & 0.609 & $60.9 \%$ \\
\hline \multicolumn{4}{|c|}{ Main percentage avalanche value } & 0.628 & $62 \%$ \\
\hline
\end{tabular}

Table 1. Shows the avalanche effect of proposed metgod

$$
\begin{aligned}
& \text { Avalanche Effect Sample } 1=\frac{4+5+5+5+4+7+6+7+6+4+4+5+5+6+4+6}{128}=\frac{83}{128}=0.648 \\
& \text { Avalanche Effect Sample } 2=\frac{4+5+5+2+4+5+4+6+6+5+6+7+4+6+6+3}{128}=\frac{78}{128}=0.609
\end{aligned}
$$

The Avalanche Effect results of the proposed method indicate that the ratio of $62 \%$ is a good ratio, where the accepted ratio is 50\% indicates the algorithm has perfect confusion and diffusion, as well as the ratio indicates that there is less repetition in the ciphertext When the blocks of plain text are almost the same. This good ratio comes from that method when it is selected the sub-magic square randomly in each encryption process. The randomness tests are important for testing the cipher text to determine if there is any deviation or biases between plaintext/cipher text bits and to ensure the random form for the cipher text. The proposed technique provides accepted results based on the NIST randomness tests. Where a P-value for a test is equal to one, and then the value will be ideal randomness. A P-value of zero refers that the value is 
completely non-random. The results tested are displayed in Table 2. The results of the randomness tests of the proposed method are acceptable for all the p-values of the statistical tests where all p-values are nearest from 1 that indicated the encrypted data are random text. Accordingly, that randomness results are acceptable and no frequency of all possible overlapping m-bit patterns across the entire sequence.

Table 2. Proposed method results of the NIST randomness tests

\begin{tabular}{clccc}
\hline & Statistical Tests & Input Size (n) & P-value Proposal method & The results \\
\hline 1 & Frequency (monobit) & 10000 & 0.873124 & Pass \\
& Test & 100000 & 0.624278 & Pass \\
& Average of P-value & & $\mathbf{0 . 7 4 8 7 0 1}$ & Pass \\
2 & Block Frequency & 10000 & 0.287645 & Pass \\
& (m=8) & 100000 & 0.634265 & Pass \\
& Average of P-value & & $\mathbf{0 . 4 6 0 9 5 5}$ & Pass \\
Approximate Entropy & 10000 & 0.876541 & Pass \\
& Test m=3 & 100000 & 0.955915 & Pass \\
& Average of P-value & 10000 & $\mathbf{0 . 9 1 6 2 2 8}$ & Pass \\
4 & Linear Complexity & 100000 & 0.447321 & Pass \\
& Test (M=100) & & 0.505732 & Pass \\
& Average of P-value & 10000 & $\mathbf{0 . 4 7 6 5 2 6}$ & Pass \\
Runs Test & 100000 & 0.289120 & Pass \\
& & 0.801208 & Pass \\
\hline
\end{tabular}

\section{CONCLUSION}

The proposed technique process the problem of repetition in the ciphertext and increase the permutation of the plain text by uses the functionality of a $3 \times 3$ magic square and the diagonal Latin square. The proposed method demonstrated the ability to process repetition in encrypted data effectively when entering similar texts in each encryption process, in addition, the method demonstrated the high randomness of the encrypted texts. The proposed was achieved by uses a magic square $12 \times 12$ to hiding encrypted data and eliminates the repetition elements. Thus, the encrypted data are substantially secure and robust against attackers based on the tests the Avalanche Effect and randomness results that shown accepted results in the ciphertext. It is recommended that research is used in the field that corresponding database encryption because the database security system needs encryption methods that not suffer from the problem of repetition data.

\section{REFERENCES}

[1] Das, Debasis, U. A. Lanjewar, and S. J. Sharma, "The Art of Cryptology: From Ancient Number System to Strange Number System," International Journal of Application or Innovation in Engineering\& Management (IJAIEM), vol. 2, no. 4, pp. 265-275, 2013.

[2] Stallings, William, "Network Security Essentials: Applications and Standards Fourth Edition," Prentice Hall, 1 Lake Street, Upper Saddle River, NJ, New Jersey, 2011.

[3] Danti, Ajit, and Rajesh Nayak, "Data Encryption by Excluding Repetitive Character in Cipher Text," International Journal Of Innovationsin Engineering And Technology (IJIET), vol. 2, no. 4, pp. 270-276, 2013.

[4] Ess, Eli, "The Magic of Magic Squares," Thesis in Mathematics Middlebury College, 2005.

[5] N. Shibiraj, Tomba," Modified Hill Cipher: Secure Technique using Latin Square and Magic Square," International Journal of Computer Sciences and Engineering, vol. 6, no. 12, pp. 315-320, 2018.

[6] P. Bartlett, "Latin Squares and Magic," Mathcamp, 2012.

[7] Gao, Lei, "Latin squares in experimental design," Michigan State University, 2005.

[8] D. I. George, J. Sai Geetha and K. Mani, "Add-on Security Level for Public Key Cryptosystem using Magic Rectangle with Column/Row Shifting," International Journal of Computer Applications, vol. 96, no. 14, pp. 38-43, 2014.

[9] Nitin Pandey, D. B. Ojha, "Secure Communication Scheme with Magic Square," Journal of Global Research in Computer Science, vol. 3, no. 12, pp. 12-14, 2012.

[10] P. K. Srinivasan, "Mathematics and Magic Square,” Magic Square, 1992.

[11] Ronald P. Nordgren, "New Constructions For Special Magic Squares," International Journal of Pure and Applied Mathematics, vol. 78, no. 2m pp. 133-154, 2012.

[12] Sesiano, Jacques, "Magic Squares: Their History and Construction from Ancient Times to AD 1600," Springer International Publishing, 2019.

[13] Ali, Nada Hussein M., and Suaad Ali Abead, "Modified Blowfish Algorithm for Image Encryption using Multi Keys based on five S-boxes," Iraqi Journal of Science, vol. 57, no. 4C, pp. 2968-2978, 2016.

[14] Stallings, William, "Cryptography and network security: principles and practice 6 Edition,” Person Education Inc, 2014. 
[15] Madhuri, O. B. B., E. Rambabu, and M. Malijeddi, "Design and Implementation of Arithmetic Unit for GF (2m)," International Journal of Advanced Research in Computer Engineering \& Technology, vol. 1, no. 9, 2012.

[16] Welschenbach, Michael, "Cryptography in C and C++," Apress, 2006.

[17] Dharini, A., R. M. Saranya Devi, and I. Chandrasekar, "Data Security for Cloud Computing Using RSA with Magic Square Algorithm," International Journal of Innovation and Scientific Research, vol. 11, no. 2, pp. 439-444, 2014.

[18] Liu, Chenglian, et al., "A Study on the Stream Cipher Embedded Magic Square of Random Access Files," AIP Conference Proceedings, vol. 1389, no. 1, pp. 1070-1073, 2011.

[19] Umar, Shahla Uthman, "An Improved RSA based on Double Even Magic Square of order 32," Kirkuk University Journal /Scientific Studies (KUJSS), vol 12, no. 4, pp. 1-18, 2017.

[20] Abdullah, Ako Muhammad, and Roza Hikmat Hama Aziz, "New approaches to encrypt and decrypt data in image using cryptography and steganography algorithm," International Journal of Computer Applications, vol. 143, no. 4, pp. 11-17, 2016.

[21] Alhassan, John K., et al., "A Secure Method to Hide Confidential Data Using Cryptography and Steganography," International Conference on Information and Communication Technology and Its Applications (ICTA 2016), pp. 105-110, 2016.

[22] Jeena Pappachan, Jinu Baby, "Tinkerbell Maps based Image Encryption using Magic Square," International Journal of Advanced Research in Electrical, Electronics and Instrumentation Engineering, vol. 4, no. 7, pp. 6226-6232, 2015.

[23] Wu, Yue, et al., "A novel latin square image cipher," arXiv preprint arXiv: 1204.2310, 2012.

[24] Al-Hasan, Waleed S. Hasan, Muhammad Mun'im Ahmad Zabidi, and Ab Al-Hadi Ab Rahman, "A New Steganography Technique Using Magic Square Matrix and Affine Cipher," Journal of Telecommunication, Electronic and Computer Engineering (JTEC), vol. 10, no. 2-8, pp. 119-125, 2018.

[25] Tomba, I., "Add-on security using Weak Magic squares in Public Key Cryptosystem, Modified with Dummy Letters," International Journal of Scientific and Research Publications, vol. 7, no. 2, pp. 257-261, 2017.

\section{BIOGRAPHIES OF AUTHORS}
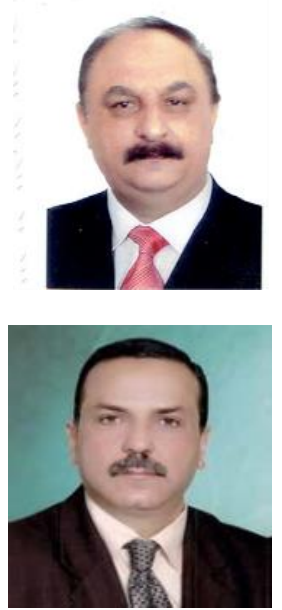

Sahab Dheyaa was born in Baghdad in 1960. He has a Ph.D. in computer science-security from Informatics Institute for Postgraduate Studies Baghdad, Iraq.has M.Sc. in computer science from the College of Science AL-Mustansiriyah University from 2003- 2005. From 1997-2000 he received his B.Sc. in Computer Science-Computer Dept. College of Education, ALMustansiriyah University. From 1980 to 1984, he received also his B.Sc in administration science in economic and administration College, AL-Mustansiriyah University. $\mathrm{He}$ is a lauctuelar in University of Information Technology and Communications. Current research interests: network security, database security, Image processing

Taha Mohammed Hasan received his BSc, MSc in computer science from Mansour University College and The University of Mustansiriyah, Baghdad, Iraq in1992 and 2006 respectively and $\mathrm{PhD}$ in computer science From Harbin Institute of Technology (HIT), Harbin, China in 2013. Currently he is an assistant professor in University of Diyala College of Sciences Computer Science Department, Iraq. His research interests include image processing, pattern recognition, cloud computing and biometrics, etc 